\title{
Robust Adaptive Control for a Class of Nonlinear Systems with Uncertainties and Input Constraints
}

\author{
Kaveh Moezzi Madani $\ddagger$ and Mansour Eslami§ \\ $\ddagger$ Dept. of Electrical Engineering, Amir Kabir University of Technology, Teheran, Iran, moezzi@ cic.aut.ac.ir \\ $\S$ Dept. of Electrical and Computer Engineering, UIC, Chicago, IL 60607, eslami@ece.uic.edu (text only)
}

\begin{abstract}
Robust adaptive controllers for multivariable nonlinear systems with fast convergence rate are designed, in spite of unwanted effects of external disturbances and input constraints. The proposed adaptive controller guarantees the local stability of the plant in the presence of input constraints, with required conditions on the upper bound for controller parameter matrices and initial conditions of the plant. These results are applied to a simulation study.
\end{abstract}

\section{Introduction}

In practical control problems, one of the major issues that arises naturally while controlling a dynamic system is the inevitable constraint on the magnitude of its control input. Hence, any input that is determined to process such a system should meet a set of desired control objectives while remaining within certain bounded limits. Ignoring these requirements when designing a controller may lead to a poor performance or even instability for the underlying system.

Samples of recent publications in direct adaptive control of linear continuous-time plants with input constraint are [4] and [10]. To the best of our knowledge for practical problems involving nonlinear systems with input constraints, there are only a few papers in the field of adaptive control of nonlinear systems such as [2], and [6]. In [6], an adaptive control scheme for nonlinear uncertain systems with actuator amplitude and the rate constraint based on Lyapunov direct method is developed. While in this paper, we design a robust direct adaptive control for multivariable nonlinear systems in the presence of uncertainties and disturbance with input saturation by extending the adaptive controller in [1] and [6], using the concept of auxiliary input in [7] and [8]. Here, we find conditions for the upper bounds of parameter matrices and initial conditions of the plant, in order to guarantee its local stability in the presence of input saturation.

\section{Robust Adaptive Controller for Nonlinear Sys- tems}

A multivariable nonlinear uncertain plant $P$ is described as follows.

$$
\begin{aligned}
\dot{x}(t)=[ & {\left[A(x(t))+\Delta A_{1}(x(t))+\Delta A_{2}(x(t))\right] x(t) } \\
& +[B(x(t))+\Delta B(x(t))] u(t)+d(t),
\end{aligned}
$$

where $A(x) \in R^{n \times n}, B(x) \in R^{n \times m}$ are state-dependent, timevarying matrices, for $n \geq m ; x \in R^{n}, x(0)=x_{0}, t \geq 0$, is directly the measurable state vector; in addition we assume that the output vector is the same as this state vector; $u(t) \in R^{m}$ is the adaptive control input vector; $d(t) \in R^{n}$ is a disturbance vector such that

$$
\|d(t)\| \leq\|d(t)\|_{\max } \triangleq \xi_{d} .
$$

In (1), $\Delta A_{1}(x) \in R^{n \times n}$ is an unstructured uncertainty; while $\Delta A_{2}(x) \in R^{n \times n}$ is a structured uncertainty which belongs to the set $D$ as follows.

$$
\begin{aligned}
D=\left\{\Delta A_{2}: R^{n} \rightarrow R^{n \times n}: \Delta A_{2}=A_{\delta}(x) \delta\left(h_{\delta}(x)\right),\right. \\
\left.x \in R^{n}, \delta(\cdot) \in \Delta\right\},
\end{aligned}
$$

with $\delta(\cdot)$ belonging to the following set

$$
\Delta=\left\{\delta: R^{l_{\delta}} \rightarrow R^{p_{\delta} \times n}:\left\|\delta\left(h_{\delta}(x)\right)\right\| \leq\left\|w\left(h_{\delta}(x)\right)\right\|\right\} .
$$

In (3), $A_{\delta}(x) \in R^{n \times p_{\delta}}, h_{\delta}(x): R^{n} \rightarrow R^{l_{\delta}}$ are known functions of the state of the plant; $\delta \in R^{p_{\delta} \times n}$ is an uncertain function which is bounded by (4); $w \in R^{p_{\delta} \times n}$ is a known function and $\Delta B(x) \in R^{n \times m}$ is an unstructured uncertainty.

The reference model for (1) is described by

$$
\dot{x}_{r}(t)=A_{r} x_{r}(t)+B_{r} r(t),
$$

and the reference input $r(t) \in R^{m}$ is bounded as

$$
\|r(t)\| \leq\|r(t)\|_{\max } \triangleq \xi_{r}
$$

In (5), $A_{r} \in R^{n \times n}$ and $B_{r} \in R^{n \times m}$ are such that the pair $\left\{A_{r}, B_{r}\right\}$ is completely controllable, and $A_{r}$ is chosen as an Hurwitzian matrix. The reference input $r(t) \in R^{m}$ has been chosen such that $x_{r}(t)$ represents the desired trajectory for the corresponding plant at time t.

Our objective is to determine the adaptive input $u(t)$ so that for all admissible $x(t)$ and $u(t)$, and a given set of initial conditions the system remains bounded. Furthermore system state $x(t)$ tracks $x_{r}(t)$ as closely as possible. To that end, first, we design the controller structure by assuming that the plant matrices are known; second, we consider the closed-loop system with the above mentioned control law. Subsequently, by using Lyapunov direct method we find the adaptation laws.

Lemma 2.1. Consider the plant $P$ in (1) without uncertainties, disturbance and any constraint on the input, but with known plant matrices $A\left(x_{p}\right)$ and $B\left(x_{p}\right)$ as follows.

$$
\dot{x}_{p}(t)=A\left(x_{p}\right) x_{p}(t)+B\left(x_{p}\right) u(t) .
$$

Then the control law

$$
u(t)=\hat{B}\left(x_{p}\right) \hat{K}_{1}\left[r+\hat{K}_{2} \hat{A}\left(x_{p}\right) x_{p}(t)\right]
$$

guarantees the convergence of the error

$$
e_{p}(t) \triangleq x_{p}(t)-x_{r}(t)
$$

to zero, if there exist gain matrices and state-dependent known matrices such as $\hat{K}_{1} \in R^{m \times m}, \quad \hat{K}_{2} \in R^{m \times s}$ $\hat{B}\left(x_{p}\right) \in R^{m \times m}$ and $\hat{A}\left(x_{p}\right) \in R^{s \times n}$ such that

$$
\begin{gathered}
B\left(x_{p}\right) \hat{B}\left(x_{p}\right) \hat{K}_{1}=B_{r}, \\
A\left(x_{p}\right)-A_{r}+B_{r} \hat{K}_{2} \hat{A}\left(x_{p}\right)=0 .
\end{gathered}
$$

Further, the solution is globally asymptotically stable. 
Proof. Substituting control law (8) in (7) and using $(10-\mathrm{a})$, we obtain the error equation as follows.

$$
\begin{aligned}
\dot{e}_{p}= & A_{r} e_{p}(t)+\left[A\left(x_{p}\right)-A_{r}+B_{r} \hat{K}_{2} \hat{A}\left(x_{p}\right)\right] x_{p}(t) \\
& +\left[B\left(x_{p}\right) \hat{B}\left(x_{p}\right) \hat{K}_{1}-B_{r}\right] r(t) .
\end{aligned}
$$

From (10) we find the dynamic error (11) as follows.

$$
\dot{e}_{p}(t)=A_{r} e_{p}(t),
$$

where $A_{r}$ is Hurwitzian, and subsequently it follows that the error which is defined in (9) approaches zero. Thus, the solution is globally asymptotically stable. Q.E.D.

In Lemma 2.1 , it is shown that there exist gain matrices $\hat{K}_{1}, \hat{K}_{2}$ and state-dependent known matrices $\hat{A}(x)$ and $\hat{B}(x)$ such that they provide perfect tracking with known plant matrices $A(x)$ and $B(x)$. Now, we find timevarying adaptive gain matrices $K_{1}(t)$ and $K_{2}(t)$ such that the system state $x(t)$ tracks $x_{r}(t)$ as closely as possible in the presence of uncertainties and disturbance.

\section{Using a control law}

$$
u(t)=\hat{B}(x) K_{1}(t)\left[r+K_{2}(t) \hat{A}(x) x(t)+u_{\text {aux }}(t)\right],
$$

in plant (1) yields

$$
\begin{aligned}
\dot{x}(t)= & A_{r} x(t)+B_{r}\left(K_{2}-\hat{K}_{2}\right) \hat{A}(x) x(t)+B_{r} u_{\text {aux }}(t) \\
& +B_{r}\left(\hat{K}_{1}^{-1}-K_{1}^{-1}\right) \hat{B}^{-1} u(t)+B_{r} r(t) \\
& +\left[\Delta A_{1}(x)+\Delta A_{2}(x)\right] x(t)+\Delta B(x) u(t)+d(t),
\end{aligned}
$$

where, $K_{1}(t) \in R^{m \times m}$ and $K_{2}(t) \in R^{m \times s}$ are time-varying gain matrices and $u_{\text {aux }}(t)$ is an auxiliary control that is designed subsequently [7] and [8]. We define the parameter and state errors $\Phi_{1}(t), \Phi_{2}(t)$ and e(t) as follows.

$$
\begin{gathered}
\Phi_{1}(t) \triangleq \hat{K}_{1}^{-1}-K_{1}^{-1}(t), \Phi_{2}(t) \triangleq K_{2}(t)-\hat{K}_{2}, \\
e(t) \triangleq x(t)-x_{r}(t) .
\end{gathered}
$$

The differential equation that represents state error defined in (15-b) with uncertainties and disturbance is as follows.

$$
\begin{aligned}
\dot{e}= & A_{r} e+B_{r} \Phi_{1}(t) \hat{B}^{-1} u(t)+B_{r} \Phi_{2}(t) \hat{A} x(t)+B_{r} u_{\text {aux }}(t) \\
& +\left[\Delta A_{1}(x)+\Delta A_{2}(x)\right] x(t)+\Delta B(x) u(t)+d(t) .
\end{aligned}
$$

In order to proceed further with our constructional method, we use direct Lyapunov approach to find adaptation control policies such that the state of the system (1) tracks that of the reference model in (5) as closely as possible in the presence of uncertainties and disturbance.

Theorem 2.1. Consider the plant $\mathrm{P}$ in (1) with unstructured uncertainty $\Delta B(x)=0$. Suppose that there exist the gain matrices $\hat{K}_{1} \in R^{m \times m}, \quad \hat{K}_{2} \in R^{m \times s}$ with $\operatorname{det} \hat{K}_{1} \neq 0$, and state-dependent known matrices $\hat{B}(x) \in R^{m \times m}, \hat{A}(x) \in R^{s \times n}$ with $\operatorname{det} \hat{B}(x) \neq 0$ such that they satisfy conditions in (10). Then the control input (13), for $u_{\text {aux }}=u_{d}+u_{l}+u_{s}+u_{p}$ such that

$u_{d}=-\left\{B_{r}^{T} P e\left\|B_{r}^{\dagger}\right\| \xi_{d}\right\} /\left\|B_{r}^{T} P e\right\|$,

$u_{l}=-S B_{r}^{T} P e$,

$u_{s}=-\left\{B_{r}^{T} P e\|P e\|\left\|w\left(h_{\delta}(x)\right)\right\|\left\|A_{\delta}\right\|\|x\|\right\} /\left\|B_{r}^{T} P e\right\|^{2}, \quad(17-\mathrm{c})$

$u_{p}=-\left\{B_{r}^{T} P e\|P e\|\left\|\Delta A_{1}\right\|_{\max }\|x\|\right\} /\left\|B_{r}^{T} P e\right\|^{2}$, and adaptation laws

$\dot{\Phi}_{1}(t)=-Q_{1} B_{r}^{T} P e u^{T} \hat{B}^{-T}$,

$\dot{\Phi}_{2}(t)=-Q_{2} B_{r}^{T}$ Pe $x^{T} \hat{A}^{T}$,

guarantees the stability of (16). Here $0<P=P^{T} \in R^{n \times n}$ is the solution of the following Riccati matrix equation.

$-\dot{P}=A_{r}^{T} P+P A_{r}-2 P B_{r} S B_{r}^{T} P+Q$,

where $\quad 0<Q=Q^{T} \in R^{n \times n}, \quad 0<S=S^{T} \in R^{m \times m}$, $0<Q_{1}=Q_{1}^{T} \in R^{m \times m}$ and $0<Q_{2}=Q_{2}^{T} \in R^{m \times m}$. The superscript $\dagger$ represents Penrose pesudo-inverse of $B_{r}$ which exists if $B_{r}^{T} B_{r}$ is a nonsingular matrix [5].

Proof. Define a positive-definite function $V_{1}$ as the Lyapunov function

$$
\begin{aligned}
V_{1}=e^{T}(t) P e(t)+\operatorname{Tr}\left\{\Phi_{1}^{T}(t)\right. & \left.Q_{1}^{-1} \Phi_{1}(t)\right\} \\
& +\operatorname{Tr}\left\{\Phi_{2}^{T}(t) Q_{2}^{-1} \Phi_{2}(t)\right\} .
\end{aligned}
$$

Taking derivative of (20) along (16) when $\Delta B(x)=0$, and for $u_{\text {aux }}=u_{d}+u_{l}+u_{s}+u_{p}$ with (17) to (19) yields

$$
\dot{V}_{1} \leq-e^{T} Q e \text {. }
$$

Thus, $\dot{V}_{1} \leq 0$, which shows (16) is stable. Q.E.D.

Remark 2.1. With respect to $K_{1}(t)$, the Lyapunov function defined in (20) is not radially unbounded. Therefore Theorem 2.1 yields local stability. In addition, it can be shown that $\dot{K}_{1}(t)=K_{1}(t) \dot{\Phi}_{1}(t) K_{1}(t)$.

In Theorem 2.1, we have assumed that the uncertainty $\Delta B(x)=0$. In the next step, we remove this condition and consider the effect of uncertainty $\Delta B(x)$ on (1).

Theorem 2.2. Consider the plant $\mathrm{P}$ in (1) with $\Delta B(x) \neq 0$. Suppose that there exist the gain matrices $\hat{K}_{1} \in R^{m \times m}$, with $\operatorname{det} \hat{K}_{1} \neq 0, \hat{K}_{2} \in R^{m \times s}$ and state-dependent known matrices $\hat{B}(x) \in R^{m \times m}, \hat{A} \in R^{s \times n}$ with $\operatorname{det} \hat{B}(x) \neq 0$, such that they satisfy the conditions in (10). If

$\sqrt{\left(V_{0}\right)}<\sqrt{\left(\lambda_{\min }\left(Q_{1}^{-1}\right)\right.}\left\{\frac{1}{\left\|\hat{K}_{1}\right\|}-\left\|B_{r}^{\dagger}\right\|\|\Delta B\|_{\max }\|\hat{B}\|_{\max }\right\},(22-\mathrm{a})$
$\|\Delta B\|_{\max }<1 /\left\{\left\|B_{r}^{\dagger}\right\|\|\hat{B}\|_{\max }\left\|\hat{K}_{1}\right\|\right\}$,

then the control input (13), for $u_{\text {aux }}=u_{l}+u_{p}+u_{a}$ such that

$u_{l}=-S B_{r}^{T} P e$,

$u_{p}=-\left[\gamma_{1}+\frac{\gamma_{2}}{\left\|B_{r}^{T} P e\right\|}+\frac{\gamma_{3}}{\left\|B_{r}^{T} P e\right\|^{2}}\right] B_{r}^{T} P e$,

$u_{a}=-\frac{B_{r}^{T} P e\|P e\|\left(\left\|w\left(h_{\delta}(x)\right)\right\|\left\|A_{\delta}\right\|+\left\|\Delta A_{2}\right\|_{\max }\right)\|x\|}{\left\|B_{r}^{T} P e\right\|^{2}}$,

with

$\beta^{-1} \triangleq 1-\left\|B_{r}^{\dagger}\right\|\|\Delta B(x)\|_{\max }\|\hat{B}(x)\|\left\|K_{1}(t)\right\|$,

$\gamma_{1}=\beta\left\|B_{r}^{\dagger}\right\|\|\Delta B(x)\|_{\max }\left\|\hat{B}(x) K_{1}(t)\right\|\|S\|$,

$\gamma_{2}=\beta\left\|B_{r}^{\dagger}\right\| \times\left\{\|\Delta B\|_{\max }\left\|\hat{B} K_{1} K_{2} \hat{A}\right\|\|x\|\right.$ $\left.+\|\Delta B\|_{\max }\left\|\hat{B} K_{1}\right\| \xi_{r}+\xi_{d}\right\}$,

$\gamma_{3}=\beta\left\|B_{r}^{\dagger}\right\|\|\Delta B\|_{\max }\left\|\hat{B} K_{1}\right\|\|P e\| \times$

$\left(\left\|w\left(h_{\delta}(x)\right)\right\|\left\|A_{\delta}\right\|+\left\|\Delta A_{2}\right\|_{\max }\right)\|x\|$ 
and adaptation laws in (18), guarantees the stability of (16). Here $\mathrm{P}, \mathrm{Q}, \mathrm{S}$ and $Q_{1 \& 2}$ are defined in Theorem 2.1. and $V_{0}$ is the initial condition of (20).

Proof. Using the derivative of (20) along (16) when $\Delta B(x) \neq 0$ and for $u_{\text {aux }}=u_{l}+u_{p}+u_{a}$ becomes

$$
\begin{aligned}
\dot{V}_{1}= & e^{T}\left(\dot{P}+A_{r}^{T} P+P A_{r}\right) e \\
& +2 \operatorname{Tr}\left\{\Phi_{1}^{T}\left(Q_{1}^{-1} \dot{\Phi}_{1}+B_{r}^{T} \operatorname{Peu}(t)^{T} \hat{B}^{-T}(x)\right)\right\} \\
& +2 \operatorname{Tr}\left\{\Phi_{2}^{T}\left(Q_{2}^{-1} \dot{\Phi}_{2}+B_{r}^{T} \operatorname{Pex}^{T} \hat{A}^{T}(x)\right)\right\} \\
& +\left(2 P B_{r} S B_{r}^{T} P+2 e^{T} P B_{r} u_{l}\right) \\
& +2 e^{T} P\left\{\left(\Delta A_{1}(x)+\Delta A_{2}(x)\right) x(t)\right. \\
& +\Delta B(x) \hat{B}(x) K_{1}\left(r+K_{2} \hat{A}(x) x+u_{l}+u_{p}+u_{a}\right) \\
& +d(t)\}+2 e^{T} P B_{r}\left(u_{p}+u_{a}\right) .
\end{aligned}
$$

Substituting (18) and (23-a) in (26) yields.

$$
\begin{aligned}
& \dot{V}_{1}\left[e(t), K_{1}(t), K_{2}(t)\right] \\
& \quad=2 e^{T} P\left\{\left(\Delta A_{1}(x)+\Delta A_{2}(x)\right) x(t)\right. \\
& \quad+\Delta B(x) \hat{B}(x) K_{1}\left(r+K_{2} \hat{A}(x) x+u_{l}+u_{p}+u_{a}\right) \\
& \quad+d(t)\}+2 e^{T} P B_{r}\left(u_{p}+u_{a}\right) \\
& \quad \leq-e^{T} Q e+2\left\|e^{T} P\right\|\left(\left\|w\left(h_{\delta}(x)\right)\right\|\left\|A_{\delta}\right\|+\left\|\Delta A_{2}\right\|_{\max }\right)\|x\| \\
& \quad+2\left\|e^{T} P B_{r}\right\|\left\|B_{r}^{\dagger}\right\| \times\left\{\|\Delta B(x)\|_{\max }\left\|\hat{B}(x) K_{1}\right\| \xi_{r}\right. \\
& \quad+\|\Delta B(x)\|_{\max }\left\|\hat{B}(x) K_{1}\right\|\left\|K_{2} \hat{A}(x)\right\|\|x\| \\
& \quad+\|\Delta B(x)\|_{\max }\left\|\hat{B}(x) K_{1}\right\|\|S\|\left\|B_{r}^{T} P e\right\|+\xi_{d} \\
& \left.\quad+\|\Delta B(x)\|_{\max }\left\|\hat{B}(x) K_{1}\right\|\left\|u_{\text {aux }}\right\|\right\}+2 e^{T} P B_{r}\left(u_{p}+u_{a}\right) .(27)
\end{aligned}
$$

Substituting (23) to (25) in (27) yields $\dot{V}_{1} \leq-e^{T} Q_{1} e$, if $\beta^{-1}>0$. To meet this requirement, using (20) we obtain.

$$
\left\|\Phi_{1}(t)\right\|_{\max }<\sqrt{V_{0} \lambda_{\max }\left(Q_{1}\right)} .
$$

From (22-a) and (28) we find that

$$
\left\|\Phi_{1}\right\|_{\max }<\left[1 /\left\|\hat{K}_{1}\right\|\right]-\left\|B_{r}^{\dagger}\right\|\|\Delta B\|_{\max }\|\hat{B}\|_{\max } \text {. }
$$

From (15-a) we obtain

$$
K_{1}(t)-\hat{K}_{1}=K_{1}(t) \Phi_{1}(t) \hat{K}_{1},
$$

and subsequently from norms properties and (29) we conclude that

$$
\left\|K_{1}(t)\right\|-\left\|\hat{K}_{1}\right\| \leq\left\|K_{1}(t)\right\|\left\|\Phi_{1}\right\|_{\max }\left\|\hat{K}_{1}\right\|,
$$

which yields

$$
\left\|K_{1}(t)\right\|_{\max }=\left\|\hat{K}_{1}\right\| /\left\{1-\left\|\hat{K}_{1}\right\|\left\|\Phi_{1}\right\|_{\max }\right\} .
$$

Clearly, by substituting $\left\|K_{1}(t)\right\|_{\max }$ in (32) as $\left\|K_{1}(t)\right\|$ in (24), we obtain $\beta^{-1}>0$. Inequality (22-a) yields

$$
\left\|\Phi_{1}\right\|_{\max }<1 /\left\|\hat{K}_{1}\right\|-\left\|B_{r}^{\dagger}\right\|\|\Delta B\|_{\max }\|\hat{B}\|_{\max } .
$$

Because $\|\Phi\|_{\max }>0$ and from (33) we have

$$
\left[1 /\left\|\hat{K}_{1}\right\|\right]-\left\|B_{r}^{\dagger}\right\|\|\Delta B\|_{\max }\|\hat{B}\|_{\max }>0,
$$

that yields the condition in (22-b). Q.E.D.

\section{Adaptive Control with Input Constraints}

In the preceding section, we have proposed a robust adaptive controller for a class of nonlinear systems without any constraint on the functional input which is applied to the system. Here our objective is to develop an adaptive scheme for nonlinear systems with input constraints and uncertainties.
Consider the plant $\mathrm{P}$ in (1), with uncertainties $\Delta A_{1}$, $\Delta A_{2}, \Delta B$ and disturbance $d(t)$, which are defined in Section 2 ; in addition we assume that the functional input $u(t) \in R^{m}$ is constrained by the following requirement, where $C_{i}$ is a known positive quantity.

$$
\left|u_{i}(t)\right| \leq C_{i}, \text { with } i=1, \ldots, m .
$$

We define an adaptive controller input as

$$
v(t)=\hat{B}(x) K_{1}(t)\left[r+K_{2}(t) \hat{A}(x) x(t)+u_{a u x}(t)\right],
$$

where, $K_{1}(t) \in R^{m \times m}, K_{2}(t) \in R^{m \times s}$ are time-varying matrices and $u_{\text {aux }}(t)$ is an auxiliary input. Because the system functional input $u(t)$ in (1) is bounded, we define such an input in terms of our input $v(t)$ in (36) and its corresponding error as follows.

$$
u_{i}(t)=\left\{\begin{array}{cc}
v_{i}(t), & \text { if }\left|v_{i}(t)\right| \leq C_{i}, \\
C_{i} \operatorname{sgn}\left(v_{i}(t)\right), & \text { if }\left|v_{i}(t)\right|>C_{i},
\end{array}\right.
$$

where, $i=1, \ldots, m$ and

$$
\Delta u(t) \triangleq u(t)-v(t) .
$$

Suppose, there exists the parameter matrix gain $\hat{K}_{3} \in R^{n \times q}$ and $\hat{B}_{\Delta}(x) \in R^{q \times m}$ such that

$$
B(x)=\hat{K}_{3} \hat{B}_{\Delta}(x)
$$

for the purpose of removing the effect of $\Delta u(t)$, as in [4] we generate $e_{\Delta}(t)$ from the following differential equation.

$\dot{e}_{\Delta}(t)=A_{r} e_{\Delta}(t)+K_{3}(t) \hat{B}_{\Delta}(x) \Delta u(t)$, with $e_{\Delta}(0)=0$.

By defining

$$
\Phi_{3}(t) \triangleq \hat{K}_{3}-K_{3}(t)
$$

and an augmented error $e_{1}$ as

$$
e_{1}(t) \triangleq e(t)-e_{\Delta}(t)
$$

and using (1), (15) and (41), the differential equation for the dynamical error $e_{1}(t)$ becomes

$$
\begin{aligned}
\dot{e}_{1}(t)= & A_{r} e_{1}(t)+B_{r} \Phi_{1}(t) \hat{B}^{-1} v(t)+B_{r} \Phi_{2}(t) \hat{A}(x) x(t) \\
& +\Phi_{3}(t) \hat{B}_{\Delta}(x) \Delta u(t)+\left[\Delta A_{1}(x)+\Delta A_{2}(x)\right] x(t) \\
& +B_{r} u_{\text {aux }}(t)+\Delta B(x) v(t)+\Delta B(x) \Delta u(t)+d(t) .
\end{aligned}
$$

In the next step, using Lyapunov direct method we construct the adaptation laws for parameter matrices $\Phi_{i}$ 's, which guarantee the stability of the $e_{1}(t)$ with $\Delta B=0$.

Lemma 3.1. Consider the plant $P$ in (1) with unstructured uncertainty $\Delta B(x)=0$ and bounded input $u(t)$. Suppose that there exist the gain matrices $\hat{K}_{1} \in R^{m \times m}$, $\hat{K}_{2} \in R^{m \times s}, \hat{K}_{3} \in R^{n \times q}$ with $\operatorname{det} \hat{K}_{1} \neq 0$ and state-dependent known matrices $\hat{B}(x) \in R^{m \times m}, \quad \hat{A}(x) \in R^{s \times n} \quad$ with $\operatorname{det} \hat{B}(x) \neq 0$ and $\hat{B}_{\Delta}(x) \in R^{q \times m}$ such that they satisfy conditions in (10) and (39). Then the control input (36) with functional input (37), for $u_{\text {aux }}=u_{d}+u_{l}+u_{s}+u_{p}$ such that

$u_{d}=-\left\{B_{r}^{T} P e_{1}\left\|P e_{1}\right\| \xi_{d}\right\} /\left\|B_{r}^{T} P e_{1}\right\|^{2}$,

$u_{l}=-S B_{r}^{T} P e_{1}$,

$u_{s}=-\left\{B_{r}^{T} P e_{1}\left\|P e_{1}\right\|\left\|w\left(h_{\delta}(x)\right)\right\|\left\|A_{\delta}\right\|\|x\|\right\} /\left\|B_{r}^{T} P e_{1}\right\|^{2},(44-\mathrm{c})$ $u_{p}=-\left\{B_{r}^{T} P e_{1}\left\|P e_{1}\right\|\left\|\Delta A_{1}\right\|_{\max }\|x\|\right\} /\left\|B_{r}^{T} P e_{1}\right\|^{2}$,

with adaptation laws 
$\dot{\Phi}_{1}(t)=-Q_{1} B_{r}^{T} P e_{1}(t) u^{T} \hat{B}^{-T}$,

$\dot{\Phi}_{2}(t)=-Q_{2} B_{r}^{T} P e_{1}(t) x^{T} \hat{A}^{T}$,

$\dot{\Phi}_{3}(t)=-Q_{3} P e_{1}(t) \Delta u^{T}(t) \hat{B}_{\Delta}^{T}$,

guarantees the boundedness of $e_{1}(t)$ and $\Phi_{i}$ 's. Here $\mathrm{P}$ is the solution of (19), where $0<Q=Q^{T} \in R^{n \times n}$, $0<S=S^{T} \in R^{m \times m}, \quad 0<Q_{1}=Q_{1}^{T} \in R^{m \times m}$, $0<Q_{2}=Q_{2}^{T} \in R^{m \times m}$ and $0<Q_{3}=Q_{3}^{T} \in R^{n \times n}$.

Proof. The proof is similar to Theorem 2.1 by considering the positive-definite Lyapunov function $V_{2}(t)$

$$
\begin{aligned}
V_{2}(t)= & e_{1}^{T}(t) P e_{1}(t)+\operatorname{Tr}\left\{\Phi_{1}^{T}(t) Q_{1}^{-1} \Phi_{1}(t)\right\} \\
& +\operatorname{Tr}\left\{\Phi_{2}^{T}(t) Q_{2}^{-1} \Phi_{2}(t)\right\}+\operatorname{Tr}\left\{\Phi_{3}^{T}(t) Q_{3}^{-1} \Phi_{3}(t)\right\},
\end{aligned}
$$

and substituting (44) and (45) into its derivative. Q.E.D.

In the next step, we develop a control law such that it stabilizes the error defined in (42) with bounded inputs and $\Delta B \neq 0$. Also we assume that, $\Delta A_{1 \& 2}=0$ and $d(t)=0$. The results can be extended to the plant (1).

Theorem 3.1. Consider the plant $\mathrm{P}$ in (1) with unstructured uncertainty $\Delta B(x) \neq 0, \Delta A_{1 \& 2}=0, d(t)=0$ and bounded input $u(t)$. Suppose that there exist the gain matrices $\hat{K}_{1} \in R^{m \times m}, \hat{K}_{2} \in R^{m \times s}, \hat{K}_{3} \in R^{n \times q}$ with $\operatorname{det} \hat{K}_{1} \neq 0$, and state-dependent known matrices $\hat{B}(x) \in R^{m \times m}$, $\hat{A}(x) \in R^{s \times n}$ with $\operatorname{det} \hat{B}(x) \neq 0$ and $\hat{B}_{\Delta}(x) \in R^{q \times m}$ such that they satisfy conditions in (10) and (39). If the conditions (22) are held, where $V_{0}=V_{2}(0)$ and $V_{2}(0)$ is the initial condition of Lyapunov function in (46), then the control input (36), with functional input (37) for $u_{\text {aux }}=u_{l}+u_{p 1}+u_{\Delta}$ that $u_{l}$ and $u_{p 1}$ satisfying

$u_{l}=-S B_{r}^{T} P e_{1}(t)$,

$u_{p 1}=-\left[\left\|\alpha^{*}\right\| \gamma_{1}+\frac{\gamma_{2}}{\left\|B_{r}^{T} P e_{1}(t)\right\|}\right] B_{r}^{T} P e_{1}(t)$,

where, $\beta^{-1}=1-\left\|\alpha^{*}\right\|\left\|B_{r}^{\dagger}\right\|\|\Delta B(x)\|_{\max }\|\hat{B}(x)\|\left\|K_{1}(t)\right\|, \gamma_{1}$ is defined in (25) and

$$
\begin{aligned}
& \gamma_{2}=\beta\left\|B_{r}^{\dagger}\right\|\|\Delta B\|_{\max } \begin{array}{r}
\left\|\hat{B} K_{1}\right\|\left\|\alpha^{*}\right\| \times\left\{\left\|K_{2} \hat{A}\right\|\|x\|\right. \\
\left.+\xi_{r}+\left\|B_{r}^{\dagger}\right\|\|\Delta B\|_{\max }\|C\|\right\},
\end{array} \\
& \alpha_{i}^{*}= \begin{cases}1, & \text { if } \Delta u_{i}(t)=0, \\
0, & \text { if } \Delta u_{i}(t) \neq 0,\end{cases}
\end{aligned}
$$

where, $i=1, \ldots, m$, and $\alpha^{*}=\operatorname{diag}\left[\alpha_{i}^{*}\right]$,

$u_{\Delta}=-\left\{B_{r}^{T} P e_{1}\left\|B_{r}^{\dagger}\right\|\|\Delta B\|_{\max }\|C\|\right\} /\left\|B_{r}^{T} P e_{1}\right\|$,

and $C=\left(I_{m}-\alpha^{*}\right)\left[C_{1}, \ldots, C_{m}\right]^{T}$ with adaptation laws in (45), guarantees the boundedness of $e_{1}$ and $\Phi_{i}$ 's. Here, P, $\mathrm{Q}, \mathrm{S}$ and $Q_{1 \& 2 \& 3}$ are defined in Lemma 3.1.

Proof. Using Lyapuov function in (46), the proof is similar to that of Theorem 2.2. Q.E.D.

\subsection{Improving Transient Response}

Obviously, the behavior of the system in its transient trajectory is important, because the signals may exceed some limits that cause the steady state errors or even instability of the system. We can see this effect in systems with input constraint, while the input fluctuates very fast and will take large values in the transient time. One way to improve the performance of the system is to decrease the transient response time for the system by increasing its convergence speed. Convergence speed of two or more different Lyapunov designs can be compared by a positive value $\eta_{i}=-\dot{V}_{i} / V_{i}, i=1,2, \cdots$ [3]. Therefore design A has a faster convergence rate than design B if $\eta_{A}>\eta_{B}$.

In [7] and [8], using an auxiliary input the lower bound of $\eta$ has been maximized. In this design we maximize the lower bound of $\eta$, using parameter matrix $\Phi$, in order to analyze the robustness of the system with constrained input. To proceed further, consider plant $\mathrm{P}$ in (1) without uncertainties $\Delta A_{1}, \Delta A_{2}, \Delta B$ and disturbance $d(t)$. Using control law (36) and (37) with $u_{\text {aux }}(t)=0$ we obtain the dynamic equation for $e_{w} \triangleq x-x_{r}$ as follows.

$\dot{e}_{w}(t)=A_{r} e_{w}(t)+B_{r} \Phi_{1}(t) \hat{B}^{-1} u(t)+B_{r} \Phi_{2}(t) \hat{A}(x) x(t)$.

Here, we define $e_{2}(t)=e_{w}-e_{\Delta}$ that yields

$$
\begin{aligned}
\dot{e}_{2}(t)= & A_{r} e_{2}(t)+B_{r} \Phi_{1}(t) \hat{B}^{-1} v(t)+B_{r} \Phi_{2}(t) \hat{A}(x) x(t) \\
& +\Phi_{3}(t) \hat{B}_{\Delta}(x) \Delta u(t),
\end{aligned}
$$

Defining

$$
\begin{gathered}
\Phi(t)=\left[\Phi_{1}(t), \Phi_{2}(t)\right]^{T}, \\
\omega(t)=\left[\left(\hat{B}^{-1} v(t)\right)^{T},(\hat{A}(x) x(t))^{T}\right]^{T},
\end{gathered}
$$

the dynamic error (49) becomes

$$
\dot{e}_{2}(t)=A_{r} e_{2}(t)+B_{r} \Phi^{T}(t) \omega(t)+\Phi_{3} \hat{B}_{\Delta} \Delta u(t) .
$$

Lemma 3.2. Consider the differential equation (52) and suppose that there exist the gain matrices $\hat{K}_{1} \in R^{m \times m}$, $\hat{K}_{2} \in R^{m \times s}, \hat{K}_{3} \in R^{n \times q}$ with $\operatorname{det} \hat{K}_{1} \neq 0$, and state-dependent known matrices $\hat{B}(x) \in R^{m \times m}, \hat{A}(x) \in R^{s \times n}$ with $\operatorname{det} \hat{B}(x) \neq 0$ and $\hat{B}_{\Delta}(x) \in R^{q \times m}$ such that they satisfy conditions in (10) and (39). Then the adaptation law,

$\dot{\Phi}(t)=-Q_{4} \omega(t) e_{2}^{T}(t) P B_{r}-m(t) Q_{4} U \Phi(t)$,

and $\dot{\Phi}_{3}(t)=-Q_{3} P e_{2}(t) \Delta u^{T}(t) \hat{B}_{\Delta}^{T}$ with

$m(t)>-\left\{\lambda_{\min }(Q)\left\|e_{2}\right\|^{2}\right\} / \operatorname{Tr}\left\{\Phi^{T} U \Phi\right\}$,

guarantees the boundedness of $e_{2}$ and $\Phi$. Here, $0<P=P^{T} \in R^{n \times n}$ such that

$$
A_{r}^{T} P+P A_{r}=-Q,
$$

where $\quad 0<Q=Q^{T} \in R^{n \times n}, \quad 0<Q_{4}=Q_{4}^{T} \in R^{m \times m}$, $0<U=U^{T} \in R^{m \times m}$ and $0<Q_{3}=Q_{3}^{T} \in R^{n \times n}$.

Proof. Derivative of the positive-definite Lyapunov function

$$
V_{3}(t)=e_{2}^{T} P e_{2}+\operatorname{Tr}\left\{\Phi^{T} Q_{4}^{-1} \Phi\right\}+\operatorname{Tr}\left\{\Phi_{3}^{T} Q_{3}^{-1} \Phi_{3}\right\},
$$

along (52), upon substituting (53) \& (54) completes the proof.

$$
\dot{V}_{3}(t)=-e_{2}^{T} Q e_{2}-2 m(t) \operatorname{Tr}\left\{\Phi^{T} U \Phi\right\} \leq 0 .
$$

\subsection{Robustness Analysis}

In this section, we provide the conditions where the solution of the system (49) with adaptation law (54) is bounded. 
Theorem 3.2. The differential equation (52) with adaptation law (53) and assumptions in Lemma 3.2. has bounded solution if,

$$
\begin{aligned}
& x^{T}\left(t_{0}\right) P x\left(t_{0}\right)<\lambda_{\min }(P)[\rho / \tau]^{2}, \\
& \sqrt{\left(V_{0}\right)}<\sqrt{\lambda_{\min }\left(Q_{4}^{-1}\right)} \times\left[q_{0} \rho-2\left\|P_{b}\right\| \xi_{r} \tau\right] / \\
& {\left[2\left\|P_{b}\right\|\left\|\hat{B}^{-1}\right\|_{\max } C_{n} \tau+2\left\|P_{b}\right\|\|\hat{A}\|_{\max } \rho\right],} \\
& \rho=C_{0} s_{0} \lambda_{\text {min }}\left(\hat{B}^{-1} \hat{B}^{-T}\right) \lambda_{\text {min }}\left(\hat{K}_{1}^{-1} \hat{K}_{1}^{-T}\right) \lambda_{\text {min }}\left(P_{b} P_{b}^{T}\right) \text {, } \\
& \tau=\left\|P_{b}\right\|\left\|\hat{B}^{-1}\right\|_{\max }\left\|\hat{K}_{1}^{-1}\right\|\|S\| \times \\
& \left|2\left\|P_{b}\right\|\left\|\hat{K}_{2}\right\|\|\hat{A}\|_{\max }-q_{0}\right| \text {. }
\end{aligned}
$$

Here, $q_{0}=\lambda_{\min }(Q), s_{0}=\lambda_{\min }(S), P_{b}=P B_{r}, C_{0}$ is the minimum component of the vector $C=\left[C_{1}, \ldots, C_{m}\right]^{T}, C_{n}$ is the norm-2 of $C ; 0<P=P^{T} \in R^{n \times n}$ such that (55) is held; $\quad 0<Q=Q^{T} \in R^{n \times n}, \quad 0<Q_{4}=Q_{4}^{T} \in R^{m \times m}$, $0<S=S^{T} \in R^{m \times m}$ and $V_{0}$ is the initial condition of the Lyapunov function defined in (56).

Proof. From (14), (15) and (38) the plant equation without any uncertainty becomes

$$
\begin{aligned}
\dot{x}(t)=A_{r} x(t)+B_{r} \Phi_{1}(t) \hat{B}^{-1} u(t) & +B_{r} \Phi_{2}(t) \hat{A}(x) x(t) \\
& +B_{r} r(t)+B(x) \Delta u(t) .
\end{aligned}
$$

We provide the proof for the boundedness of $x(t)$ by considering the cases when

$\left|v_{i}(t)\right| \leq C_{i}$, for all $i=1, \ldots, m$ and $\Delta u_{i}=0$,

$\left|v_{i}(t)\right|>C_{i}$, for all $i=1, \ldots, m$, and $\Delta u_{i} \neq 0$,

$\left|v_{i}(t)\right|>C_{i}$, for some $i=1, \ldots, m$.

First, consider (61-a), by defining a positive-definite Lyapunov function $W=\frac{1}{2} x^{T} P x$, the derivative of $W$ along (60) becomes.

$\dot{W}=-\frac{1}{2} x^{T} Q x+x^{T} P_{b} \Phi_{2} \hat{A} x+x^{T} P_{b} r+x^{T} P_{b} \Phi_{1} \hat{B}^{-1} v$

Clearly, $\|\Phi\| \leq\|\Phi\|_{\max }$ and subsequently, from (50) $\left\|\Phi_{1}\right\| \leq\|\Phi\|_{\max }$ and $\left\|\Phi_{2}\right\| \leq\|\Phi\|_{\max }$, therefore $\dot{W} \leq 0$, if

$$
\|x\|>\frac{2\left\|P_{b}\right\|\left\|\hat{B}^{-1}\right\|_{\max }\|\Phi\|_{\max } C_{n}+2\left\|P_{b}\right\| \xi_{r}}{q_{0}-2\left\|P_{b}\right\|\|\hat{A}\|_{\max }\|\Phi\|_{\max }} .
$$

Second, consider (61-b), the derivative of $W$ along (60) when $\Delta u \neq 0$ becomes.

$$
\begin{aligned}
\dot{W}=-\frac{1}{2} x^{T} Q x & +x^{T} P_{b} \hat{K}_{2} \hat{A} x \\
& +\sum_{i=1}^{m}\left(x^{T} P_{b} \hat{K}_{1}^{-1} \hat{B}^{-1}\right)_{(1, i)} C_{i} \operatorname{sgn}\left(v_{i}(t)\right),
\end{aligned}
$$

where, $\left(x^{T} P_{b} \hat{K}_{1}^{-1} \hat{B}^{-1}\right)_{(1, i)}$ is $(1, i)^{t h}$-element of $\left(x^{T} P_{b} \hat{K}_{1}^{-1}\right.$ $\left.\hat{B}^{-1}\right) \in R^{1 \times m}$. Now, consider three cases for the signs of $v_{i}(t)$.

Case $\mathbf{A}, \quad \operatorname{sgn}\left(v_{i}\right)=\operatorname{sgn}\left(x^{T} P_{b} \hat{K}_{1}^{-1} \hat{B}^{-1}\right)_{(1, i)}$, for all $i=1, \ldots, m$ : Here, (64) becomes

$\dot{W}=-\frac{1}{2} x^{T} Q x+x^{T} P_{b} \hat{K}_{2} \hat{A} x+\sum_{i=1}^{m} C_{i}\left|x^{T} P_{b} \hat{K}_{1}^{-1} \hat{B}^{-1}\right|_{(1, i)}(65)$

Since $\left|v_{i}\right|>C_{i}$, we have
$\sum_{i=1}^{m}\left(x^{T} P_{b} \hat{K}_{1}^{-1} \hat{B}^{-1}\right)_{(1, i)} v_{i}>\sum_{i=1}^{m}\left|\left(x^{T} P_{b} \hat{K}_{1}^{-1} \hat{B}^{-1}\right)_{(1, i)}\right| C_{i}$, (66-a)

then

$-\frac{1}{2} x^{T} Q x+x^{T} P_{b} \hat{K}_{2} \hat{A} x+\sum_{i=1}^{m}\left(x^{T} P_{b} \hat{K}_{1}^{-1} \hat{B}^{-1}\right)_{(1, i)} v_{i}>$

$-\frac{1}{2} x^{T} Q x+x^{T} P_{b} \hat{K}_{2} \hat{A} x+\sum_{i=1}^{m}\left|\left(x^{T} P_{b} \hat{K}_{1}^{-1} \hat{B}^{-1}\right)_{(1, i)}\right| C_{i} \cdot(66-\mathrm{b})$

Adding and subtracting terms $x^{T} P_{b} K_{2} \hat{A} x$ and $x^{T} P_{b} r$ to the left-hand side of (66-b) and using (63) yields

$0>-\frac{1}{2} x^{T} Q x+x^{T} P_{b} \Phi_{2} \hat{A} x+x^{T} P_{b} r+x^{T} P_{b} \Phi_{1} \hat{B}^{-1} v$

$>-\frac{1}{2} x^{T} Q x+x^{T} P_{b} \hat{K}_{2} \hat{A} x+\sum_{i=1}^{m} C_{i}\left|x^{T} P_{b} \hat{K}_{1}^{-1} \hat{B}^{-1}\right|_{(1, i)}$

which, shows $\dot{W}<0$.

Case $\mathbf{B}, \operatorname{sgn}\left(v_{i}\right)=-\operatorname{sgn}\left(x^{T} P_{b} \hat{K}_{1}^{-1} \hat{B}^{-1}\right)_{(1, i)}$, for all $i=1, \ldots, m$ : Here, (64) becomes

$\dot{W}=-\frac{1}{2} x^{T} Q x+x^{T} P_{b} \hat{K}_{2} \hat{A} x-\sum_{i=1}^{m} C_{i}\left|x^{T} P_{b} \hat{K}_{1}^{-1} \hat{B}^{-1}\right|_{(1, i)}$

which is negative semi-definite if

that yields (58-a).

$$
\|x\|<\rho / \tau,
$$

Case C, $\operatorname{sgn}\left(v_{i}\right)=\operatorname{sgn}\left(x^{T} P_{b} \hat{K}_{1}^{-1} \hat{B}^{-1}\right)_{\left(1_{1} i\right)}$, for some $i=1, \ldots, m$ and $\operatorname{sgn}\left(v_{i}\right)=-\operatorname{sgn}\left(x^{T} P_{b} \hat{K}_{1}^{-1} \hat{B}^{-1}\right)_{(1, i)}$ for others: Using (63) and (68), it can be shown $\dot{W}<0$ herein.

From (63) and (68) we construct the region $A$ such that $\dot{W} \leq 0$, and thus $x$ remains bounded. The region can be written as follows.

$A \triangleq\left\{\frac{2\left\|P_{b}\left|\left\|\mid \hat{B}^{-1}\right\|_{\max }\|\Phi\|_{\max } C_{n}+2\left\|P_{b}\right\| \xi_{r}\right.\right.}{q_{0}-2\left\|P_{b}\right\|\|\hat{A}\|_{\max }\|\Phi\|_{\max }}<\|x\|<\frac{\rho}{\tau}\right\}$

yielding

$\|\Phi\|_{\max }<\left[\frac{q_{0} \rho-2\left\|P_{b}\right\| \xi_{r} \tau}{2\left\|P_{b}\right\|\left\|\hat{B}^{-1}\right\|_{\max } C_{n} \tau+2\left\|P_{b}\right\|\|\hat{A}\|_{\max } \rho}\right]$.

Therefore we obtain (58-b).

Third, two cases (61-a) and (61-b) cover (61-c), and here we omit the details. Hence, $x(t)$ is bounded due to the conditions in (58). Further $e_{2}, \Phi$ and $u(t)$ are bounded [9].

\section{Numerical Example}

The results of the preceding sections are now applied to the following nonlinear uncertain system given by [1],

$\dot{x}_{1}=\delta_{1} x_{1}(t)+\left(1+\delta_{2}\right) x_{2}(t)$,

$\dot{x}_{2}=-\beta x_{1}(t)+\varepsilon\left(\alpha-x_{1}^{2}\right) x_{2}(t)+\frac{b+\delta_{3}}{1+x_{1}^{2}+x_{2}^{2}} u(t)$,

where, $\alpha, \beta, \varepsilon, b, \delta_{i} \in R$. are unknown with $\delta_{i} \in[-1,1]$. The reference model is given by

$$
\dot{x}_{r}=\left[\begin{array}{cc}
0 & 1 \\
-A_{1} & -A_{2}
\end{array}\right] x_{r}+\left[\begin{array}{l}
0 \\
1
\end{array}\right] r(t),
$$


where, $A_{1}=100$ and $A_{2}=10$. We assume that the control input is constrained with $C_{1}=12$, when the maximum of the control input in unconstrained case is $u_{\max }=26$. We choose

$$
\begin{aligned}
& \hat{A}(x)=-\left[\begin{array}{ccc}
1 & 0 & 0 \\
0 & 1 & x_{1}^{2}
\end{array}\right]^{T}, \hat{B}=1+x_{1}^{2}+x_{2}^{2}, \\
& \hat{K}_{1}=\frac{1}{b}, \hat{K}_{2}=\left[-\beta+A_{1}, \quad \varepsilon \alpha+A_{2}, \quad-\varepsilon\right], \\
& \Delta B=\left[0, \frac{\delta_{3}}{1+x_{1}^{2}+x_{2}^{2}}\right]^{T}, A_{\delta}=[1,0]^{T}, \delta=\left[\delta_{1}, \delta_{2}\right], \\
& \hat{B}_{\Delta}(x)=1 /\left[1+x_{1}^{2}+x_{2}^{2}\right], \hat{K}_{3}=[0, b]^{T} .
\end{aligned}
$$

Applying the results developed in this paper with the following set of parameters $\alpha=1, \beta=1, \varepsilon=2, b=3$, $\delta_{i}=1$ and initial conditions $x(0)=[0,0]^{T}, K_{1}(0)=0.1$ and $K_{2}(0)=[0,0,0]^{T}$, we can show that the results reported in the literature are substantially improved. We define $e_{1}(t)=x_{1}-x_{r 1}$ and $e_{2}(t)=x_{2}-x_{r 2}$ as state error trajectories. The state error trajectories and control signal versus time, in both saturated and unsaturated cases, which, only auxiliary input is used to remove the effect of uncertainties, and the oscillations between plant state and desired state are shown in Fig. 1. The state error trajectories and input control signal versus time for both saturated and unsaturated cases are shown in Fig. 2. Here, we apply $m(t)$ in addition to auxiliary input to the system, so that the fast convergence rate for both saturated and unsaturated cases is obtained, while the maximum amplitude of the controller is reduced. In addition, the error between plant state and desired reference state approaches zero, whether the input is saturated or not.
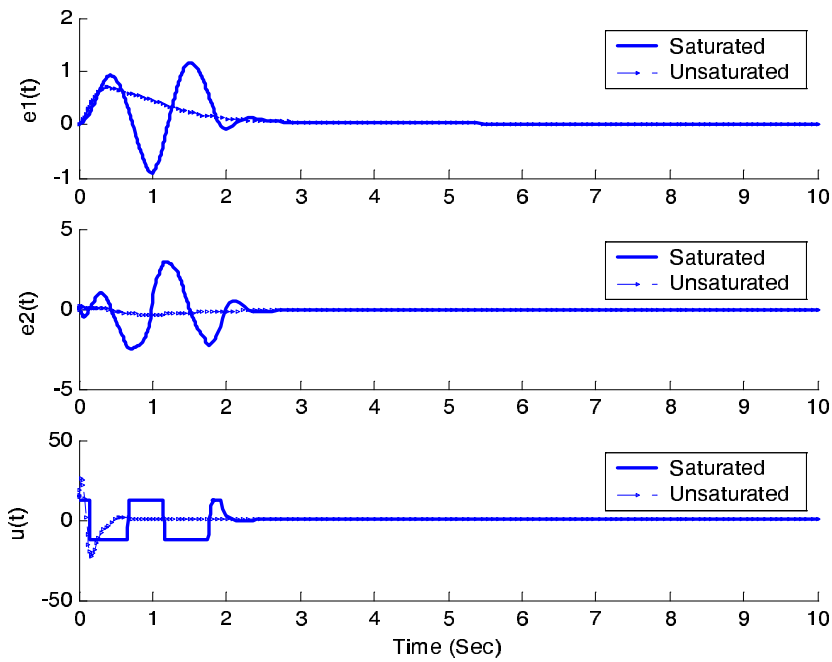

Fig. 1. Error trajectories and control input versus time, $m(t)=0$.

\section{Conclusions}

In this paper, we have considered the adaptive control of multivariable nonlinear plants in the presence of uncertainties and disturbance with input constraints. The convergence rate of the adaptive controller is improved and it is shown that the controller in the presence of saturation is locally stable with prior knowledge of the upper bounds for parameter matrices and initial conditions.
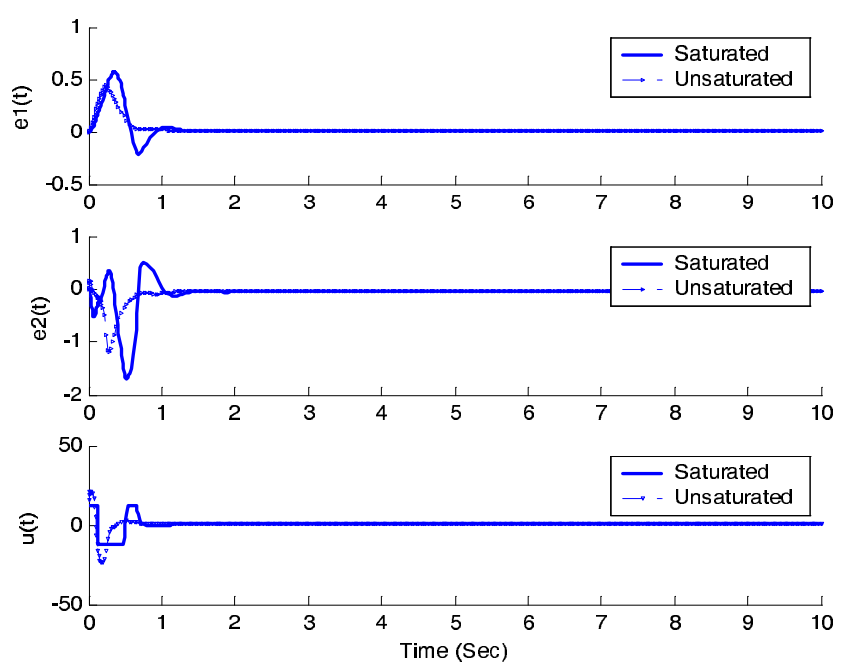

Fig. 2. Error trajectories and control input versus time, $m(t) \neq 0$.

\section{References}

[1] W. M. Haddad, T. Hayakawa and V. Chellaboina, "Robust Adaptive Control for Nonlinear Uncertain Systems", Automatica, vol. 39, pp. 551-556, 2003.

[2] R.A. Hess and S.A. Snell, "Flight control design with rate saturating actuators", AIAA J. Guid. Contr. Dyn., vol. 20, pp. 90-96, 1997.

[3] R.E. Kalman and J.E. Bertram, "Control system analysis and design via the 'Second Method' of Lyapunov", ASME Trans. J. Basic Eng., pp. 371-393. June 1960.

[4] S. P. Karason and A. M. Annaswamy, "Adaptive control in the presence of input constraints," IEEE Trans. Automat. Contr., vol. 39, pp. 2325-2330, 1994.

[5] Y.D. Landau, Adaptive Control - The Model Reference Approach. New York: Marcel Dekker, Inc., 1979.

[6] A. Leonessa, W. M. Haddad and T. Hayakawa, "Adaptive Tracking for Nonlinear Systems with Control Constraints", in Proc. ACC, pp. 1292-1297, 2001.

[7] K.Y. Lim, "Modern Control Manipulator Design", Ph.D. Thesis, Department of Electrical Engineering, State Univ. of New York at Stony Brook, Dec. 1985.

[8] K. Y. Lim and M. Eslami, "Robust Adaptive Controller Designs for Robot Manipulator Systems", IEEE J. of Robotics and Auto., vol. RA-3, No. 1, pp. 54-66, 1987.

[9] K. S. Narendra and A. M. Annaswamy, Stable Adaptive Systems. Englewood Cliffs, N. J., Prentice-Hall, 1989.

[10] C. Zhang and R. Evans, "Continues Direct Adaptive Control with Saturation Input Constraint", IEEE Trans. Auto. Cont., vol 39, No. 8, pp. 1718-1722, 1994.

Acknowledgment. Mansour Eslami acknowledges greatly the generous support provided by the Ali Mohammad Eslami's Foundation in Teheran while visiting Amir Kabir University. 